\title{
Gerechtigkeit und Moralismus
}

\section{Treffen die Einwände des politischen Realismus gegenüber Rawls zu?}

\section{Justice and Moralism}

\section{Are the Objections of Political Realists to Rawls Justified?}

\section{Amadeus Ulrich, Frankfurt am Main}

Zusammenfassung: Der neue politische Realismus erkennt in John Rawls einen Erzfeind. In jüngeren Debatten scheint oft evident zu sein, dass gerade Eine Theorie der Gerechtigkeit exemplarisch für einen Moralismus sei, der die politische Wirklichkeit verzerre. Doch die Sache ist kompliziert. In diesem Aufsatz blicke ich zurück auf sein Frühwerk im Lichte dieser Kritik. Dabei geht es mir um vier Einwände: dass Rawls' Idealtheorie (i) kein Ratgeber für das politische Handeln und ideologisch verblendet sei; (ii) Macht und ihre Legitimierbarkeit nicht überzeugend konzipiere; (iii) die Bedeutung politischer Konflikte und Uneinigkeit über den Inhalt der Gerechtigkeit unterschätze; und (iv) verkenne, dass eine wahrlich politische Theorie auf die Geschichte eingehen müsse. Wie stichhaltig sind diese Vorwürfe und was fördern sie zutage? Der Aufsatz zeigt, dass selbst Rawls' erstes Hauptwerk nicht so einfach eines Moralismus überführt werden kann, wie es auf den ersten Blick den Anschein hat. Damit meine ich nicht, dass die Einwände allesamt keinen Biss hätten. Doch werden die Rechtfertigungslasten der Rawls'schen Gerechtigkeitstheorie nicht immer gebührend berücksichtigt; und manche Konterpunkte sind gerade bei wichtigen philosophischen Weggabelungen nicht ausreichend mit argumentativer Substanz untermauert. Wer einen Anti-Moralismus verficht, wofür es gute Gründe gibt, sollte weiterhin das konstruktive Gespräch auch mit dem frühen Rawls suchen und auf die internen Spannungen sowie realistischen Konzessionen seines Egalitarismus achten.

Schlagwörter: Moralismus, Politischer Realismus, John Rawls, Gerechtigkeit, Konflikt 
Abstract: The new political realism sees John Rawls as an archenemy. In recent debates, it often seems to be evident that especially A Theory of Justice is exemplary for a moralism that distorts political life as we know it. Yet the matter is convoluted. In this essay, I look back at his early work through the lens of this critique, which I break down into four objections: that Rawls's ideal theory (i) is not a guide to political action and blinded by ideology; (ii) cannot convincingly conceptualize power and its legitimation; (iii) underestimates the significance of political conflicts and disagreement about the content of justice; and (iv) fails to recognize that a truly political theory requires historical reflection. How valid are these charges, and what do they bring to light? The essay shows that even Rawls's first major work cannot be as easily convicted of moralism as it appears at first blush. By this, I do not mean that the objections all lack cogency. But the justificatory burdens of Rawls's theory of justice are not always duly considered; and some counterpoints, especially at important philosophical forks in the path, are not sufficiently backed up with argumentative substance. Those who advocate an anti-moralism, for which there are good reasons, should continue to seek a constructive conversation with the early Rawls and pay heed to the tensions within and realistic concessions of his egalitarianism.

Keywords: Moralism, Political Realism, John Rawls, Justice, Conflict

John Rawls beginnt Eine Theorie der Gerechtigkeit ${ }^{1}$ bekanntlich mit dem Satz, dass die Gerechtigkeit die erste und damit wichtigste Tugend sozialer Institutionen sei $(T G, 19)$. Jene Rechte, die auf ihr als einem Grundwert beruhen, sind demnach weder verhandelbar, noch dürfen sie aufgrund von Interessenabwägungen verletzt werden. Zwar räumt er ein, dass die intuitive Annahme eines Vorrangs der Gerechtigkeit wohl zu stark formuliert ist. Dennoch macht er es sich zur Aufgabe, sie vernünftig zu begründen. Folgt man Bernard Williams und Raymond Geuss, entpuppt sich Rawls bereits zu Beginn seines ersten Hauptwerks als weltentrückter Moralist, der mit seiner vermeintlich „politischen“ Theorie die komplexe Realität des politischen Lebens verschleiert und verzerrt. In Outside Ethics (im Folgenden $O E$ ) schreibt Geuss etwa, Rawls habe mit der „Fantasiewelt“ (Never-neverland) seines

$1 \quad$ Ich nutze die Abkürzung Theorie und verwende für die Schriften von Rawls folgende Siglen: Eine Theorie der Gerechtigkeit - TG; Gerechtigkeit als Fairneß - GaF; Geschichte der politischen Philosophie - GPP; und Politischer Liberalismus - PL. Ich beziehe mich auf die revidierte Fassung von Theorie, die der deutschen Übersetzung zugrunde gelegen hat. 
frühen Modells die politische Philosophie resolut in die falsche Richtung gelenkt $(O E, 33,39)$.

Um einige Einwände dieser beiden scharfsinnigen Denker gegenüber Rawls' früher Gerechtigkeitskonzeption geht es mir. Williams und Geuss haben einen neuen „Realismus“ in der politischen Philosophie inspiriert, der viele Gesichter hat. Dessen Anhänger:innen tendieren dazu, die Idee einer rein moralischen Normativität abzulehnen, die die Handlungssphäre der Politik überwölbt, ihr gewissermaßen vorgeordnet ist. ${ }^{2}$ Sie propagieren ein von der Moraltheorie unabhängigeres, empirisch informiertes politisches Denken, dessen Maßstäbe der Kritik in den konstitutiven Eigenschaften der Politik, wie wir sie kennen, fest verankert sind. Die politische Philosophie sollte, heißt es, ihr Augenmerk statt auf idealistische Konzeptionen distributiver Gerechtigkeit verstärkt auf Begriffe wie Autorität, Macht und ihre Legitimierbarkeit sowie die Bedingungen der Stabilität in von Konflikten über das Richtige, Gute und Wahre immerfort geplagten Gesellschaften richten (Forrester 2019, 276; Larmore 2020, 45f.). Ziel ist es, durch die Setzung eines neuen Startpunkts normativer Reflexion inmitten der Irrungen und Wirrungen politischer Wirklichkeit, gepaart mit einem Sinn für Geschichte, die Disziplin davor zu bewahren, angewandte Ethik zu betreiben und sich weiterhin auf die illusorische Suche nach einem archimedischen „Blickwinkel der Ewigkeit“" $(T G, 637)$ zu begeben.

Die realistische Denkschule in der politischen Philosophie ist, wie Geuss in Reality and Its Dreams (im Folgenden $R D$ ) schreibt, eine „geräumige Kirche" $(R D, 50){ }^{3}$ Doch ist nicht immer klar, gegen was genau darin gepredigt wird. ${ }^{4}$ Deshalb greife ich mir einige konkrete Kritikpunkte von Williams und Geuss heraus, die Rawls als einen moralistischen Erzfeind auserkoren haben, und frage: Wie stichhaltig sind die Einwände gegenüber Theorie? Kann der Blick zurück auf sein Frühwerk im Lichte der realistischen Kritik Stärken und Schwächen mancher Argumentationslinien zutage fördern, die für einen Blick nach vorne weiter wichtig sind? Dabei muss auch

2 Was dies jedoch genau bedeutet, ist umstritten. Die Unterscheidung zwischen einer distinkten „politischen“ versus „moralischen“ Normativität wurde vermehrt kritisiert, etwa von Leader Maynard und Worsnip (2018).

3 Gute Überblicke geben Galston (2010); McQueen (2018); Rossi und Sleat (2014); Rossi (2019); Sleat (2013).

4 Zu möglichen Variationen eines „Anti-Moralismus“, vgl. die lehrreiche Analyse und Kritik von Estlund (2020, Kap. 3). 
Rawls' „politische Wende“ (Weithman 2010) bedacht werden, deren Facetten ich hier aber nur skizzieren kann.

Mir scheint, dass selbst der frühere Gang von Rawls' Argumentation nicht so einfach eines Moralismus überführt werden kann. Denn oft werden entweder sein interpretatives Vorgehen und die tatsachensensitiven Bürden der Rechtfertigung der Gerechtigkeit als Fairness nicht gebührend berücksichtigt oder manche Konterpunkte nicht ausreichend mit argumentativer Substanz untermauert. Dieser Aufsatz stellt aber keine reine Apologie dar. Was es heißt, in theoretischen Kontexten auf fälschliche Weise zu moralisieren, ist eine wichtige und in der politischen Philosophie relativ selten untersuchte Frage (Buddeberg 2020, 185), und es lassen sich durchaus manche Passagen und Gedankenfiguren aus Theorie als Beispiele anführen. Jedoch sollte eine Moralismuskritik statt apodiktisch so präzise wie möglich sein und ihre eigenen normativen Voraussetzungen offenlegen, ohne sich dabei in Widersprüche und Inkonsistenzen zu verstricken.

Im Folgenden gehe ich zunächst näher auf den Kerneinwand ein, Rawls habe politische Theorie als angewandte Ethik betrieben und skizziere vier mögliche Stoßrichtungen einer anti-moralistischen Kritik an seinem Ansatz. In der sich auf dieser Grundlage entfaltenden Diskussion kommen einige realistische Konzessionen und Spannungen innerhalb seines ersten Hauptwerks ans Licht, die heute, gerade angesichts der vermehrt konstatierten Krise der Demokratie, weiterhin relevant sind.

\section{Politische Theorie als moralistisches Blendwerk?}

Geuss revoltiert, wie er in Kritik der politischen Philosophie (im Folgenden $K P P)^{5}$ sagt, gegen die Idee, dass die „Arbeit der Ethik“ zuerst vollendet werden kann, um zu einer idealen Theorie zu gelangen, ohne dabei groß auf empirische Begebenheiten in Gesellschaften, die Motivationsstruktur ihrer Mitglieder oder die pluralistischen Genealogien politisch-moralischer Konzepte achten zu müssen $(K P P, 22)$. Die Politik erscheint in der Vorstellung, an die sich der Moralismus klammert, prioritär als ein Werkzeug zur Durchsetzung von höherstufigen, invarianten Moralgrundsätzen und Idealen, die von der Komplexität politischen Handelns und den Widrigkeiten, denen es sich ausgesetzt sieht, bereinigt sind. Letztlich, so heißt es, strebt der Moralismus eine

5 Das Original ist das 2008 publizierte Philosophy and Real Politics. Geuss hat den Text für die deutsche Übersetzung überarbeitet und ergänzt. 
Überwindung der Politik an, weil er deren objektive Gesetzmäßigkeiten als auch Phänomene der Macht missachtet und ignoriert, was Menschen in gegebenen Umständen tatsächlich bewegt (ebd., 23). In gekonnt hyperbolischem Stil beklagt sich Geuss deshalb über die normative Wende in der politischen Philosophie in den 1970ern. Statt kontextabhängige Bedürfnisse, spezifische Institutionen sowie Kulturformen zu studieren, hätte das Gros der Philosophie vor allem im Anglo-Amerikanischen Raum einen Eskapismus praktiziert: „Witch doctors“ wie Rawls sind ihm zufolge in einen vermeintlich rein normativen Bereich geflüchtet, ,der in relativer Isolation von den schmutzigen Tatsachen der Geschichte und einer empirischen Untersuchung menschlicher Gesellschaft studiert werden könnte" ( $R D$, viii; Übers. A.U.).

Auch Williams hat sich in seinem Spätwerk, wenngleich weniger wortgewaltig, über ein verbreitetes Moralisieren in der politischen Philosophie beschwert. In seinem posthum publizierten In the Beginning Was the Deed (im Folgenden $I B W D$ ) nennt er neben dem Utilitarismus die Theorie der Gerechtigkeit als Fairness als Hauptkontrahenten eines realistischen Ansatzes, der einem distinkten politischen Denken eine größere Autonomie gewährt (IBWD, 3). Ihm ist bewusst, dass Rawls den politischen Charakter seiner Theorie eines modernen liberalen Staates, geprägt von Wertepluralismus und tiefen Konflikten, später zu betonen bestrebt war (Williams 2014, 326-332); doch habe Rawls stets an der Grundidee einer Priorität der Moral vor dem Politischen festgehalten $(I B W D, 2)$. Theorie und $P L$ eine ein „strukturelles Modell“ des politischen Moralismus, demzufolge eine Theorie die moralischen Bedingungen der Koexistenz innerhalb von Herrschaftsverhältnissen bestimmt und strikt festlegt, wann Ausübungen von Macht gerecht sind (ebd., if.). Rawls' politische Theorie sei angewandte Ethik und missverstehe die Praxis der Politik als Verwirklichung vor-politischer moralischer Verpflichtungen.

Dies umfasst eine Annahme über die Identität eines genuin politischen Denkens, das sich um ein Verständnis des eigentümlichen Bereichs des Politischen und der ihm inhärenten Kriterien der Machtkritik bemüht. Der Realismus will mit ungetrübtem Blick die Idee eines legitimen politischen Verhältnisses auf den Begriff bringen. Moralistische Ansätze würden wiederum die Hobbes'sche Wahrheit über den Ursprungsgrund politischer Institutionen und Praktiken verdrängen: die Unausweichlichkeit von moralischen Uneinigkeiten und sozialen Konflikten, die stets kanalisiert und reguliert werden müssen, um Stabilität, Schutz, Sicherheit und kooperative Verhältnisse zu gewährleisten. Mit der Interpretation eines umkämpften Herrschaftskontexts, seiner Geschichte und internen Legitimitätsbedingun- 
gen sollte in einer politischen Theorie begonnen werden, statt ihr aus Furcht vor dem Schreckgespenst des Werterelativismus die Zwangsjacke einer Platonischen oder Kantischen Moral überzustülpen ( $R D$, Kap. 2).

Daraus folgt keine Resignation gegenüber der Realität. Geuss und Williams sind nicht der weltfremden Ansicht, dass die praktische Politik ein moralfreier Raum sei, in dem es eigennützigen Akteur:innen vornehmlich um den Erhalt und die Erweiterung von Macht und ihre materiellen Interessen gehe. Sie suchen vielmehr nach einer Position zwischen Moralismus und Realpolitik. Geuss schreibt etwa, er behaupte nicht, dass „alle moralischen Überlegungen unbedingt aus der Politik ausgeschlossen sein“ müssen (KPP, 99). Verteidigt wird ein „nicht-fundamentalistisches“ (IBWD, 37), kontextsensibles und „anti-essentialistisches“ (Geuss 2010, 420) Denken, das tatsächliche Beweggründe sowie Bedürfnisse, Klagen und Ansprüche von Menschen in Betracht zieht: Die Kritik des politischen Lebens soll aus ihm selbst heraus erwachsen und sich der historischen Kontingenz ihrer normativen Grundlagen bewusst sein, statt auf vor-politische Moralvorstellungen reduziert zu werden.

Nun trifft es zu, dass Rawls nie von der Behauptung zurückgewichen ist, dass auch die politische Konzeption der Gerechtigkeit „natürlich“ eine moralische sei $(P L, 76)$. Doch äußert sich Williams in IBWD nur spärlich zur Frage, wie diese Aussage genau zu deuten ist und wo er selbst die Grenzen einer wohlverstandenen politischen Ethik zieht. Das Ganze wird noch verkompliziert, indem Williams einräumt, dass der Legitimitätsgrundsatz, für den er argumentiert, als ein moralischer aufgefasst werden könne, der jedoch der Politik nicht vorgeordnet sei (IBWD, 4f.). Dieser Imperativ besagt grob, dass die politische Aufgabe, soziale Kooperation zu gewährleisten, auf akzeptable Weise gelöst werden muss, sodass sie gegenüber den Betroffenen rechtfertigbar ist. Einige sind der Ansicht, dass der Rawls'sche Moralismus vor diesem Hintergrund keineswegs evident ist (Gledhill 2012; Jubb 2014; Thomas 2017; Sangiovanni 2008a). Doch könnte der Eindruck entstehen, dass, während der Moralismusvorwurf gegen das Spätwerk zur Debatte steht, er zumindest bei Theorie auf der Hand liegt, und sich die politische Philosophie auch deshalb aus dem Bann des ersten Hauptwerks gelöst hat. Doch mangelt es an genaueren Untersuchungen, wie viel Biss die Einwände vor allem gegen Theorie haben, die laut Williams und Geuss paradigmatisch für einen Moralismus sein müsste. ${ }^{6}$

6 Vgl. jedoch den wichtigen Aufsatz von Freyenhagen und Schaub (2010), in dem Geuss' Kritik differenziert verteidigt wird. Siehe auch Schaub (2012). 
Der Vorwurf, dass die Politik dahingehend evaluiert wird, inwiefern sie abstrakten moralischen Werten und Idealen entspricht, ist allgemein und ambig. Im Folgenden geht es mir konkret um die Analyse von vier antimoralistischen Kritikpunkten gegenüber Rawls. Diese besagen, (i) dass seine Konzeption nicht handlungsleitend sei und der politischen Wirklichkeit mit ideologischem Denken einen Zerrspiegel vorhalte; (ii) politische Macht und ihre Legitimierbarkeit nicht überzeugend konzipiere; (iii) die Bedeutung von Konflikten und moralischen Uneinigkeiten über den Inhalt der Gerechtigkeit in modernen demokratischen Gesellschaften unterschätze; und (iv) ein Bewusstsein der historischen Kontingenz politisch-moralischer Werte, Überzeugungen sowie Doktrinen vermissen lasse. Manche dieser Bemängelungen treffen nicht ins Schwarze oder sind überspitzt, meist aber dennoch erhellend: Diskutiert man sie kritisch, lassen sich wichtige Thesen und Trugschlüsse von Theorie beleuchten.

\section{Ideale, Intuitionen und Ideologie}

Eine Theorie der Gerechtigkeit sollte erläutern, inwiefern, wenn überhaupt, die Norm der Machbarkeit erfüllt ist und die politischen und sozialen Auswirkungen, die sich aus einer Umsetzung der Prinzipien ergäben, erwogen werden. Man könnte meinen, dass die realistische Kritik an Rawls sich vornehmlich auf diese Probleme der Praktikabilität eines Ideals fokussiere (Galston 2010, 387). Der Punkt ist jedoch nicht nur die zu bezweifelnde Umsetzbarkeit der Gerechtigkeitsgrundsätze, sondern die angebliche Verdrängung der Anwendungsverhältnisse politischen Handelns und Urteilens auf der prinzipiellen Ebene (Rossi und Sleat 2014, 690; Rossi 2019).

Geuss zufolge muss selbst ein minimalistischer Realismus in der politischen Philosophie einem Verständnis gegebener politischer Verhältnisse zuträglich sein: „Eine ,ideale Theorie“ ohne Kontakt zur Realität ist [...] kein Ratgeber für das Handeln“ (KPP, 128). Das Projekt der wohlgeordneten Gesellschaft sei zum Scheitern verurteilt, weil Rawls nicht darlege, wie sein normatives Modell umgesetzt werden soll. Doch dieser Einwand verengt den Zweck der politischen Philosophie zu rasch auf die Beratung im Hier und Jetzt. Bemerkenswert ist, dass es laut Rawls gegen eine Konzeption der Gerechtigkeit spräche, würden aufgrund moralpsychologischer Grundsätze und sozialer Tatsachen, die den Parteien im Urzustand auf allen Stufen des Arguments bekannt sind, Menschen keine Motivation entwickeln, sich ihr gemäß zu verhalten $(T G, 161)$. Politische Philosophie ist aber nicht „bloße Politik“ 
(Rawls 1992b, 332) und Gerechtigkeit keine strategische Frage (TG, 261). Warum sollten normative Reflexionen ohne Anspruch auf Wirkung wertlos sein, wenn es Gründe zur Annahme gibt, dass sich die Verhältnisse ändern könnten? Bietet die Geschichte nicht reichlich Material, das uns hoffen lässt, dass idealistisches Denken Fortschritte anstoßen kann, die einst nicht für vorstellbar gehalten worden sind (Estlund 2020, 84, Kap. 13)? Es ist zu bezweifeln, dass die Sinnhaftigkeit einer politischen Theorie stets davon abhängt, dass sie als Ratgeber für das Handeln fungiert. Geuss scheint dies mitunter anzunehmen, ohne die normativen Voraussetzungen, die seiner Kritik am Moralismus zugrunde liegen, sonderlich transparent zu machen (Larmore 2020, 73, Fn. 5; Schaub 2012, 22).

In $P L$ heißt es, die politische Philosophie entziehe sich allgemein nicht dem gesellschaftlichen Leben und der Welt und beanspruche auch nicht, unabhängig von „Traditionen politischen Denkens und Handelns die Wahrheit zu erkennen“ $(P L, 117)$. Das gilt auch für Theorie. Die Kritik, dass Rawls' Ansatz keinen kognitiven Kontakt zur Realität habe und beanspruche, „frei von der Kontaminierung durch die Tatsachen der Geschichte, Psychologie, Ökonomie, Soziologie und Politikwissenschaft zu sein“ (OE, 33; Übers. A.U.), führt in die Irre. Dabei gerät nicht nur sein komplexes rechtfertigungstheoretisches Vorgehen aus dem Blick, sondern auch, dass Rawls, was er allerdings erst in seinem Spätwerk genauer herausgearbeitet hat ( $G a F$, Teil IV), etwa zwischen der kritikwürdigen Idee eines kapitalistischen „Wohlfahrtsstaates“ und einer zu bejahenden „Demokratie mit Eigentumsbesitz“ unterschieden hat (TG, 308; Thomas 2017). Letztere ist seines Erachtens nebst eines gleichwertigen liberal-sozialistischen Regimes eine mögliche Realisierung der Gerechtigkeit als Fairness; und die jeweilige Umsetzung hängt von den konkreten Bedingungen, Traditionen und Institutionen einer Gesellschaft ab.

Rawls beschreibt den Urzustand als angemessene Ausgangssituation, weil moralisch willkürliche Tatsachen mit dem „Schleier des Nichtwissens Unwissenheit“ verdeckt werden, die einer fairen Entscheidung über Grundsätze sozialer Zusammenarbeit aufgrund von Interessenkalkulationen und zufälligen Machtstellungen entgegenstünden. Die Gründe für die fiktiven Bedingungen, unter denen Argumente darüber, wie Vorteile und Bürden zum allseitigen Vorteil zu verteilen sind, artikuliert werden, sollen weithin anerkannt und damit schwach und der Urzustand frei von strittigen moralischen Eigenschaften sein: „Eine Gerechtigkeitsvorstellung muß aufgrund der uns bekannten Bedingungen des menschlichen Lebens gerechtfertigt sein, oder sie ist es überhaupt nicht“ $(T G, 494)$. Dieser wichtige Satz steht im dritten 
Teil von Theorie. Dort geht es unter anderem darum, inwiefern eine Gesellschaft sich nahezu zwangsfrei aus sich selbst heraus stabilisieren könnte, wenn ihre Grundstruktur gemäß einer Konzeption der Gerechtigkeit reguliert wird und Menschen diesem formativen Einfluss ausgesetzt sind. Um dies zu beurteilen, müssen die psychologischen und sozialen Konsequenzen bedacht werden, die sich aus der Regulierung durch Prinzipien ergäben, wären sie öffentlich und allgemein anerkannt. Das ist in einer „rein normativen“ $(R D, 17)$ Sphäre nicht möglich. Es ist wichtig, zu betonen, dass der Urzustand eine vermittelnde Funktion hat als politikethisches Verfahren, für das Werte und Tatsachen herangezogen werden, die selbst nicht konstruiert sind (Sangiovanni 2014; 2016). Um der Zirkularität zu entgehen, muss der Prozedur eine Reflexion vorausgehen und folgen, die die rationale Übereinkunft unter hypothetischen Bedingungen vernünftig rechtfertigen kann: Der eigentliche Begründungsdiskurs vollzieht sich, wie Rainer Forst erläutert, „,vor und nach den Überlegungen im Urzustand“, der „zugleich Mittel und Gegenstand intersubjektiver Reflexion" ist (Forst 2007, 143f.). Die Begründungsmethode des Überlegungsgleichgewichts ist dabei flexibler und offener, als es die realistische Kritik der angeblichen Weltfremdheit von Rawls' nicht-fundamentalistischem Ansatz vermuten lässt: Eine politische Moraltheorie muss ihm zufolge „die Freiheit haben, nach Belieben kontingente Annahmen und allgemeine Tatsachen heranzuziehen " $(T G, 70)$.

Im Urzustand sind Tatsachen über funktional ausdifferenzierte Gesellschaften sowie Grundfragen sozialer Organisation, die Gesetze menschlicher Psychologie, die Grundzüge ökonomischer Theorie als auch „politische Fragen“ und damit die „Anwendungsverhältnisse der Gerechtigkeit“ bekannt, auf die ich im vierten Abschnitt eingehe (ebd., 160f.). Der Einwand, Rawls behandle „die Moral“ als vorrangig vor „dem Politischen“, ist eine Nebelkerze; beide Sphären sind auf komplexe Weise verknüpft. Aber es zeigt sich, wie angedeutet, dass auf dieser Ebene der Begründung des Urzustands die realistische Kritik ansetzen muss: an der Auffassung, was die Rechtfertigungsbedingungen der Konstruktion sind, die Rawls teils aus dem politischen Leben rational zu rekonstruieren meint. Wie erläutert, attackiert der Realismus eine Form der Herleitung moralischer Ideale, bei der konstitutive Eigenschaften politischen Handelns und Urteilens unberücksichtigt bleiben. Rawls hätte jedoch gegen diese Forderung nach normativer Kompatibilität nichts einzuwenden, geht er doch davon aus, „daß das richtige Ordnungsprinzip für irgendetwas von der Eigenart dieses Etwas abhängt“ (ebd., 47). 
Geuss dürfte hier erwidern, dass Rawls verkenne, wie voraussetzungsvoll die moralischen Ausgangspunkte sind, auf die er sich verlässt, und betonen, dass ein „wohlverstandener Realismus“ einen Gegensatz zum ideologischen politischen Denken bilde (Geuss 2010, 429). Dies führt zu einem stichhaltigeren Vorwurf: dass die Wirklichkeit, die Rawls auf Grundlage der Ideen des Gerechtigkeitssinns und wohlüberlegter Urteile zu beschreiben beabsichtigt, um die Bedingungen des Urzustands zu begründen, ideologisch durch Machtverhältnisse verzerrt sein könnte. Rawls ist der Ansicht, dass wir die Bedingungen, die zur Konkretisierung des Urzustands führen, bereits akzeptieren oder, falls nicht, durch philosophische Argumente „vielleicht“ dazu gebracht werden können $(T G, 39)$. Laut Geuss ist es jedoch eine „schlechte Idee, eine politische Philosophie auf unsere Eingebungen zu gründen“ (KPP, 126). Der moralische Gesichtspunkt könnte eine Rationalisierung unwürdiger Verhältnisse sein. Rawls erkläre nicht, aus welcher Quelle die moralischen Alltagsurteile und Empfindungen ihre Kraft und Plausibilität schöpfen, ob sie historisch oder soziologisch veränderlich sind und welche Rolle ihnen in der Gesellschaft zukommt (ebd., 98f.). Folglich übersehe er die möglichen Auswirkungen der Herrschaftsverhältnisse auf seine Theorie; sein idealtheoretisches Vorgehen sei vom Status quo schlimmstenfalls korrumpiert. Für Geuss sind Machtverhältnisse derweil „so offenkundig wichtig, dass eine politische Philosophie, die sie systematisch ausblendet, automatisch als ideologieverdächtig gelten muss“" (Geuss 2010, 428).

Dies rückt die Rechtfertigungsbedürftigkeit des Urzustands als Verfahren ins grelle Licht. Doch ist die Kritik, dass der moralische Blickwinkel eine Rationalisierung bestehender verwerflicher Machtverhältnisse sein könnte, überzogen, weil sie selbst eine hohe Begründungslast trägt und zudem eine Hypothese über die Möglichkeit moralischen Wissens umfassen könnte, die philosophischer Begründung bedarf. Es ist ja nicht so, dass sich Rawls einfach auf die moralischen Alltagsüberzeugungen verlassen und die Fixpunkte als unumstößlich darstellen würde (TG, 38). Man sollte sich, schreibt er, „so wenig wie möglich unmittelbar auf seine wohlüberlegten Urteile“, bei denen die „Gefahr des Irrtums“ bereits gering ist, stützen (ebd., 61, 67). Ferner ist er sich der Probleme bewusst, die damit einhergehen, dass die vertragstheoretische Rechtfertigung der Gerechtigkeit von metakonstruktivistischen Grundsätzen abhängt, die er jedoch nirgends als moralische Tatsachen bezeichnet. Doch ebenso ist sich Rawls im Klaren darüber, dass die Grundstruktur einen erheblichen Einfluss darauf hat, was für Bedürfnisse und Ziele ihre Subjekte entwickeln: Sie bestimmt teilweise, „was für Men- 
schen sie sein wollen und was für Menschen sie sind“ (ebd., 292). Hier ließe sich dann möglicherweise in der Tat erwidern: Wenn es zutrifft, dass auch jene normativen Überzeugungen, auf die er rekurriert, sich in einer ungerechten, nichtidealen Gesellschaft herausgebildet haben könnten, dann liegt der Korrumpierungsvorwurf nahe (Freyenhagen und Schaub 2010, 463).

Doch Rawls bemerkt selbst, dass es scheinen könnte, „als spreche der Einfluß des Gesellschaftssystems auf die menschlichen Bedürfnisse und das menschliche Selbstbild entscheidend gegen die Vertragstheorie“ (TG, 293). Die moralischen Alltagsurteile müssen deshalb reiflicher Überlegung standhalten können und Rationalitätskriterien genügen, denen sich jede vernünftige Urteilsfindung zu beugen hat, und in ein kohärentes Gleichgewicht gebracht werden (Kersting 2001, 133): „Rechtfertigungsgründe liegen nicht an der Oberfläche“ (TG, 631). Hier ist zu beachten, dass Rawls der Ansicht ist, dass sich allgemeine menschliche Bedürfnisse nach Primärgütern tatsachengetreu bestimmen lassen und dass er sich auf „repräsentative Theorien aus der Tradition der Moralphilosophie“ (ebd.) beruft, die eine Übereinkunft darüber darstellen, welche Gerechtigkeitsvorstellungen sich als vernünftig und brauchbar erwiesen haben. Und eine seiner überzeugendsten Grundüberlegungen in Theorie ist, dass unsere Lebenschancen und politischen Einflussmöglichkeiten nicht von Umständen abhängen sollten, für die wir nicht verantwortlich sind, wie etwa unser Schicksal hinsichtlich der Verteilung natürlicher Gaben oder die soziale Schicht, in die wir geboren werden (ebd., §17): „Gerecht oder ungerecht ist die Art, wie sich die Institutionen angesichts dieser Tatsachen verhalten“ (ebd., 123). Insofern sollte auch niemand durch die Wahl der Grundsätze der Gerechtigkeit „aufgrund natürlicher oder gesellschaftlicher Gegebenheiten bevorzugt oder benachteiligt werden“ (ebd., 36). Ist das Ideologie?

Gewiss, Rawls' Beharren auf der Idealtheorie als notwendiger Bedingung, um systematisch unsere nichtideale Welt, in der wir mit tiefen und dringlichen Ungerechtigkeiten wie rassistischen und geschlechtsbasierten Diskriminierungen und Unterdrückungen sowie eklatanten sozialen Ungleichheiten zu kämpfen haben, kritisieren zu können, mag zu strikt sein. Es ist auch nicht einfach von der Hand zu weisen, dass er in Theorie nicht ausreichend eruiert, inwiefern die wohlabgewogenen Urteile darüber, was faire Entscheidungsbedingungen sind, vom Gesellschaftssystem verzerrt sein und Unregelmäßigkeiten unterliegen könnten. Ich werde auf die Schwierigkeit, dass konstruktivistische Konzeptionen der Gerechtigkeit von Voraussetzungen abhängen, die sie nicht selbst hervorbringen können, zurückkommen. 
Zuvor will ich mich in den nächsten beiden Abschnitten näher mit der Kritik befassen, dass Rawls ein irreführend idealisierendes Bild der Politik zeichne, weil er selbst politische Plattitüden $(I B W D, 13)$ ausklammere und die normative Priorität der sozialen Stabilität als Grundbedingung legitimer Herrschaft unter tiefen Konfliktbedingungen verkenne.

\section{Maschinen und Staatsschiffe: Rawls zu politischer Macht}

Eine der größten Mankos der Rawls'schen politischen Philosophie ist laut Geuss, dass sie sich nirgends gründlich mit Macht befasst (KPP, 123; Wolin 2004, 539). Zweifellos trifft es zu, dass der Begriff in Theorie kaum diskutiert wird. Es sei jedoch bedacht, dass sich Rawls auf die Grundstruktur als Hauptgegenstand der Gerechtigkeit bezieht, auf jene sozialen und politischen Institutionen also, die Rechte und Pflichten ihrer Mitglieder festlegen und dabei ihre Lebenschancen, das heißt, „was sie werden können und wie gut es ihnen gehen wird“, tiefgreifend beeinflussen $(T G, 23)$. Eine Institution ist demnach ein öffentliches Regelwerk, das „Ämter und Positionen bestimmt mit ihren Rechten und Pflichten, Machtbefugnissen und Schutzzonen“ (ebd., 74; Herv. A.U.). Das politische System sowie die wirtschaftlichen und sozialen Umstände legen fest, wie Güter produziert werden und wer worauf einen Anspruch hat. Jede Grundstruktur schafft ein Möglichkeitsfeld zur Reproduktion und zum Erhalt von Macht, den Verlauf des individuellen Lebens bestimmen zu können. Die Existenz von Machtungleichheit löst, weil sie aus kontingenten gesellschaftlichen und natürlichen Umständen erwächst, die Privilegien reproduzieren, einen Rechtfertigungsbedarf aus. Stets sind wir Teil eines Systems, das beeinflusst, was wir denken, begehren und tun. Will sagen: Der Schwierigkeit einer durch Strukturen und Naturlotterie konstituierten ungleichen Verteilung von Macht, die etwa Hobbes $(1984,66)$ als die Fähigkeit eines Menschen bestimmt, ein zukünftiges Gut mit den gegenwärtig verfügbaren Ressourcen zu erlangen, ist sich Rawls bewusst. Das Problem des Mangels der Thematisierung der Macht und der Frage ihrer Legitimierung in Theorie muss anders verortet werden.

Im Leviathan heißt es auch, dass die erste Aufgabe des Staates darin bestehe, die „Sicherheit“ der politischen Gemeinschaft zu gewährleisten, womit nicht nur die Erhaltung des Lebens, sondern „auch alle anderen Annehmlichkeiten des Lebens“ gemeint sind (Hobbes 1984, 255). An diesen Gedanken hat Williams angeknüpft. Er benennt als „erste politische Frage“, wie die Bedingungen sozialer Kooperation, Vertrauen, Sicherheit und Schutz 
gewährleistet werden können (IBWD, 3; KPP, 37). Nach Williams ist eine Hauptfunktion legitimer politischer Machtausübung, diese Werte zu verwirklichen und Übel, die rationale Furcht erregen, zu verhindern - eine immerwährende Aufgabe. Wie sie gelöst wird, hängt von den Einzelheiten eines kulturellen und politischen Kontextes ab. Ordnung und allgemeine Furchtfreiheit sind notwendige Voraussetzungen für andere politische Güter.

Doch darf die Antwort auf die erste politische Frage nicht selbst zum Problem werden. Für Williams besteht eine Universalie politischer Normativität darin, dass Macht allein nichts rechtfertigt $(I B W D, 5)$. Er argumentiert für einen Basic Legitimation Demand (BLD). Ein illegitimes von einem legitimen Herrschaftsverhältnis lasse sich in Bezug auf die Art, $o b$ und wie die erste politische Frage beantwortet werde, unterscheiden. Die Lösung muss, so Williams, akzeptabel sein; soll heißen, dass jene, die die Autorität beanspruchen, über Zwangsmacht verfügen zu dürfen, jedem betroffenen Subjekt eine Rechtfertigung geben müssen, warum es gute Gründe gibt, sich dem Recht und öffentlichen Entscheidungen zu fügen (ebd., 4f.). Politische Herrschaft bedarf demnach stets einer Legitimierungsgeschichte. Williams argumentiert, dass Gruppen innerhalb eines Staates nicht radikal benachteiligt werden dürfen; sie hätten allen Grund zu revoltieren, weil die Politik qua der Bedingungen eines legitimen politischen Verhältnisses ihnen gegenüber nicht hält, was sie verspricht. Wenn eine Gruppe eine andere terrorisiere und exkludiere, hätten wir es nicht mit Politik, sondern mit „erfolgreicher Beherrschung" (ebd., 5) zu tun. ${ }^{7}$ Die rechtfertigungsbedürftige politische Macht darf dabei, wie er ausführt, ihre Rechtfertigung nicht selbst produzieren, den Legitimitätsglauben politischer Subjekte an die Zwangsbefugnis nicht erzwingen. Dies nennt Williams das Critical Theory Principle (CTP). ${ }^{8}$

Diese hier kursorisch dargestellte Vorstellung politischer Legitimität ist, in moralischer Hinsicht, minimalistisch. Der Schwerpunkt liegt auf der Verhinderung von Machtmissbrauch und Grausamkeit. Nun, die Grundidee des CTP scheint mir auch bei Rawls angelegt zu sein. Denn er schreibt: „All-

7 Notabene: Das ist eine dichte Auffassung der Politik als evaluativer Kategorie, die nicht alle Vertreter:innen des neuen politischen Realismus teilen.

8 Bemerkenswert ist, wie schwer es sein kann, herauszufinden, ob die Machtverhältnisse tatsächlich kausal für einen Legitimitätsglauben verantwortlich sind und was die kritische Kraft einer solchen Erkenntnis wäre (Williams 2002, Kap. 9). Zudem ist es vorstellbar, dass sich in einer Gesellschaft Vorstellungen von legitimen politischen Praktiken und Institutionen zwangsfrei herausbilden und dennoch problematisch sind (Estlund 2020, 59f.). 
gemein ist anerkannt, daß erzwungene Versprechen von Anfang an nichtig sind“ (TG , 379). Außerdem: „Doch ganz ähnlich sind ungerechte gesellschaftliche Verhältnisse eine Art Zwang, ja Gewalt, und die Zustimmung zu ihnen ist nicht bindend“ (ebd.). Die Einsicht, dass Macht ihre Rechtfertigung nicht durch unvermittelten Zwang selbst fabrizieren darf, ist, denke ich, eines der wohlbedachten Urteile, die den Urzustand rechtfertigen; die Zufälligkeit der Drohvorteile soll den Inhalt der Gerechtigkeit nicht verzerren (ebd., 165). Indes ist unklar, inwiefern Grundsätze wie der BLD und das CTP konsistent als von einer vor-politischen moralischen Normativität unabhängig gedacht werden können: Welcher Wert wird verwirklichkeit, wenn man in politischen Verhältnissen lebt, die aufgrund von Verfahren gerechtfertigt sind, die diese Prinzipien erfüllen (Leader Maynard und Worsnip 2018, 784)?

Es läge meines Erachtens nahe, sich etwa auf den Wert wechselseitiger Achtung zu berufen, die sich in der Bereitschaft äußert, anderen Menschen relevante Gründe zu geben, die sie, unter für das Urteilen günstigen Umständen, anerkennen könnten, sollten ihre Interessen durch (politische) Maßnahmen erheblich berührt werden $(T G, 373) .{ }^{9}$ Hier muss allerdings bedacht werden, dass Rawls nicht mit einem moralischen Begriff des Respekts beginnt, weil dieser erst unabhängig hergeleitet werden müsste und daher, wie er schreibt, „keine brauchbare Grundlage“ darstellt, um die Gerechtigkeitsprinzipien zu rechtfertigen (ebd., 636).

Dennoch ist die Kritik von Williams an Rawls, dass Theorie politischer Macht zu enge Grenzen auferlege, nicht ganz unberechtigt. Für Letzteren scheint in seinem ersten Hauptwerk der Maßstab rechtmäßigen Regierens stets die Gerechtigkeit zu sein. Ideale Gesetzgeber:innen richten ihr Denken und Handeln vor allem am Grundsatz der gleichen Freiheit für alle aus; die Gerechtigkeitstheorie begründet die gerechte Verfassung, „das Ziel des politischen Handelns“ (ebd., 249). Dass in seiner Idealtheorie die Parteien im Urzustand nicht wissen, dass jene, die sich an die Grundsätze halten sollen, unfügsam sein könnten, führt zu einer strikten Perspektive auf die Bedingungen gerechtfertigter politischer Praxis und Argumentation, die sich bestenfalls auf eine „moralische Einmütigkeit“ bezieht (ebd., 296). Das ist durchaus eine arg moralisch idealisierte Vorstellung des politischen Deliberierens und Handelns, das sich in der nichtidealen Wirklichkeit bekanntlich an ei-

9 An dieser Stelle in Theorie zitiert Rawls einen frühen Aufsatz von Williams zur Idee der Gleichheit, und darin ist dieser schöne Satz zu finden: „[A]llen ist der Versuch des Verstehens geschuldet“ (IBWD, 104; Übers. A.U.). 
ner Pluralität von Gründen und Zwecken orientieren muss - darunter der Wahlsieg. Die Annahme der vollständigen Konformität nötigt Rawls dazu, Fragen auszuklammern, die er als die „wichtigsten des politischen Lebens“ benennt, etwa eine Theorie der Strafe oder der ausgleichenden Gerechtigkeit (ebd., 387). Andere Kriterien geraten so in den Hintergrund, mit denen sich beurteilen ließe, ob die Art und Weise, wie mittels politischer Verfahren auf Grundlage eines zwingenden und positiven Rechts vernünftige Gerechtigkeitsvorstellungen implementiert werden, als legitim gelten können.

Charles Larmore bringt es auf den Punkt: „Das moralisch Beste könnte nicht politisch rechtfertigbar, das heißt, es könnte illegitim sein“ (Larmore 2020, 220, 106; Übers. A.U.). Rawls sagt wiederum, dass der wesentliche Prüfstein, um politische Verfahren zu beurteilen, „von der voraussichtlichen Gerechtigkeit des Ergebnisses“ abhängt (TG, 261). Opak bleibt, welche Grundbedingungen politische Prozeduren erfüllen müssen, etwa die gebührende Inklusion aller miteinander im Konflikt liegenden Gesichtspunkte disparater Gruppen, um als legitim zu gelten (Mason 2010, 670). Rawls bettet seine Überlegungen zum Zweck und den Effekten politischer Macht in den Kontext einer rechtsstaatlichen und modernen Demokratie. Sein Ziel ist wohlgemerkt keine umfassende empirische Theorie der Politik. In Bezug auf das politische Geschehen spricht er allerdings ab und an von einer „Maschine“, in die Repräsentant:innen und Wähler:innen ihre Auffassungen eingeben, damit sie Entscheidungen, etwa hinsichtlich der Bereitstellung öffentlicher Güter, produziert (TG, 223f.; §42). Diese sollen stets im Einklang mit einer Verfassung sein, deren Kern, wie Jürgen Habermas (1996, 65-127) beklagt hat, realen politischen Verfahren zur Willensbildung enthoben ist: Rawls verbaut sich die Möglichkeit, eine Verfassung als offenes, historisch bedingtes Projekt zu begreifen (IBWD, 15); die Bauanleitung der Maschine steht vor der verfassungsgebenden Versammlung innerhalb des Vier-Stufen-Gangs bereits teils fest, weil basale Freiheitsrechte einen Vorrang vor demokratischen Legitimationsdiskursen haben. Diese Skepsis gegenüber radikaleren Auffassungen partizipatorischer Politik äußert sich auch in dem traditionellen Bild von einem „Schiff auf See“, das Rawls nutzt, um folgenden Punkt in seiner Theorie zu verankern: Sollten Verhältnisse und Ziele erkennbar sein, die allen gleichermaßen zum Vorteil wären, spreche nichts dagegen, manchen Menschen, die „weiser und urteilsfähiger“ sind, den Kurs des „Staatsschiffes“ bestimmen zu lassen, um die Fahrgäste sicher ans Ziel zu bringen (TG, 263f.). Auch das ließe sich als eine fragwürdige Moralisierung des politischen Geschehens kritisieren. 
Wiederum trifft es nicht zu, dass er die Unausweichlichkeit des Zwangs in der politischen Sphäre negiere oder die Tatsache übersehe, dass Verfahren in der Politik komplexe Lösungsversuche andauernder Probleme in einer von immer wieder hervorbrechendem Dissens geprägten Gesellschaft sind. Für Rawls wird staatliche Macht letztinstanzlich „über ein bestimmtes geographisches Gebiet“ ausgeübt und beeinflusst die Lebenschancen der Menschen (ebd., 252). Wenige Seiten später schreibt er, dass aufgrund der Tatsache, dass in Gesellschaften stets bittere Differenzen und Übel auftreten können, gleiche Freiheiten mitunter auf die am wenigsten ungerechte Weise eingeschränkt werden müssten (ebd., 274). Darüber hinaus hebt er en passant hervor, dass Feindseligkeiten und allseitiges Misstrauen sich in einer Gesellschaft derart auswachsen können, dass sich auf manche Verfahren nicht länger verlassen werden kann (ebd., 261). Erwägungen wie diese werfen die Frage auf, was vom politischen Apparat in derart tragischen Momenten in erster Instanz gefordert ist. Rawls' idealtheoretische Konzeption politischen Handelns in einer wohlgeordneten Gesellschaft verdeckt den Blick auf die Weber'sche Einsicht in die Dilemmata und ethischen Paradoxien, mit denen sich Regierende, deren Entscheidungen den Alltag von Millionen beeinflussen können und dies oft nicht ohne moralische Kosten, konfrontiert sehen, wenn sie „das Richtige“ tun wollen (Hampshire 2000, 74; Wolin 2004, 536). Der nächste Abschnitt befasst sich näher mit dem in dieser Hinsicht relevanten Problem der Konfliktregulierung.

\section{Gerechtigkeit im Konflikt und die Hobbes'sche These}

Politische Realist:innen beanspruchen für sich, Konflikte und moralische Uneinigkeiten ernster zu nehmen als Rawls. Irrtümlicherweise halte dieser fest an der Hoffnung, dass eine übergreifende Einmütigkeit in Bezug auf Grundprinzipien der Gerechtigkeit, die selbst nicht in politischen Verfahren ausgehandelt werden müssen, möglich ist (Sleat 2013, 21, 39). Die Überzeugung, dass die politische Philosophie imstande sein soll, den Dissens, der im politischen Leben über kollektiv zu verfolgende Zwecke und die Interpretation von Werten regelmäßig hervorbricht, zu lösen, wird als absurd aufgefasst (Sangiovanni 2008b, 229).

Meist bezieht man sich auf die von Jeremy Waldron $(1999,102)$ so bezeichneten „Umstände der Politik“: Nicht nur würden wir in Gesellschaften in Fragen der Religion und des Sinns des Lebens, sondern auch im Hinblick auf die fairen Modalitäten sozialer Kooperation immerfort in Konflikt gera- 
ten. Indes gebe es in menschlichen Gruppen einen Bedarf an einer gemeinsamen Rahmenordnung, Entscheidungsverfahren oder Vorgehensweisen, obgleich stets umstritten bleiben wird, was genau getan werden sollte. Mit stabilen gerechtigkeitstheoretischen Konsensen ist in der Politik demnach nicht zu rechnen. Liberale hätten nicht ausreichend anerkannt, dass „Uneinigkeit über jene Angelegenheiten, in denen wir ihres Erachtens geteilter Ansicht sein müssen“, in der Moderne unausweichlich sei (ebd., 106; Übers. A.U.). Die politische Theorie sollte vielmehr jene Prozesse, Mechanismen und Institutionen, die eine friedvolle Koexistenz durch Konfliktregulierung ermöglichen, kritisch zergliedern und analysieren (Waldron 2016, 14f.). Auch Geuss schreibt in Bezug auf Hobbes, dass politische Übereinkommen in Gesellschaften fragil und stets potenziell in Bedrängnis seien (KPP, 35).

Nun sind die Probleme von sozialem sowie weltanschaulichem Pluralismus ein charakteristisches Element des Rawls'schen Spätwerks. In Theorie fehlt eine längere Diskussion doktrinärer Konflikte. Vom „Faktum des Pluralismus“, jenem Umstand, dass sich in der öffentlichen Kultur einer freien demokratischen Ordnung umfassende moralische, philosophische und religiöse Lehren ausbilden, die wegen ihres transzendenten Inhalts im unauflöslichen Widerstreit miteinander liegen, spricht Rawls erstmals in seinen Aufsätzen ab 1985. Ist Theorie also naiv in Bezug auf die Rolle von Konflikten in der Politik? Zu Beginn seines ersten Hauptwerks heißt es doch, eine Gesellschaft sei „charakteristischerweise von Konflikt geprägt“ $(T G, 20)$.

Betrachten wir kurz die Hume'schen „Anwendungsverhältnisse der Gerechtigkeit“. Eine Grundstruktur ist Rawls zufolge gekennzeichnet durch ein geteiltes Interesse an sozialer Zusammenarbeit, weil sie für alle ein besseres Leben ermöglicht. Doch da es Menschen nicht gleichgültig ist, wie die Früchte der Kooperation und die daraus erwachsenen Vorzüge verteilt werden, und sie sich in ihren Fähigkeiten und Eigenschaften so ähnlich sind, dass niemand allein alle anderen dominieren kann, gibt es einen Bedarf an regulativen Prinzipien (ebd., §22). Gerade weil wir unterschiedliche Lebenspläne und Vorstellungen des Guten entwickeln und auf dieser Grundlage Ansprüche an natürliche und soziale Ressourcen stellen, ist eine Gerechtigkeitskonzeption notwendig. Eine harmonische Gemeinschaft, in der es keine miteinander in Konflikt liegenden Interessen und Bedürfnisse gibt und Zwang niemals vonnöten ist, wäre ,jenseits der Gerechtigkeit“ (ebd., 316). Dabei zieht Rawls auch in Betracht, dass unser Wissen, Denken und Urteilen in der Regel mangelhaft und oft verzerrt ist und sich stets äußerst verschiedene philosophische, religiöse, politische (!) und gesellschaftliche 
Anschauungen herausbilden (ebd., 150). Er sieht ein, dass sowohl politische Meinungen als auch die Einstellungen und Interessen, die sie beeinflussen, ständig kollidieren können: „Mangelnde Einmütigkeit gehört zu den Anwendungsverhältnissen der Gerechtigkeit, denn Meinungsverschiedenheiten kommen ja ständig auch zwischen ehrlichen Leuten vor, die ganz ähnlichen politischen Grundsätzen folgen möchten“ (ebd., 253).

Er bejaht zudem die Hobbes'sche These, nach der selbst in einer wohlgeordneten Gesellschaft Zwangsmechanismen der Streitbeilegung unabdingbar sind, um die Stabilität sozialer Kooperation zu gewährleisten (ebd., 271). Ein Grund ist, dass sich Menschen auch unter den günstigsten Verhältnissen bisweilen nicht vollends vertrauen; interpersonale Konflikte und schädliches Verhalten sind immer möglich - Tendenzen, die die Bedingungen der Möglichkeit gesellschaftlicher Zusammenarbeit erheblich erschüttern können. Rawls ist insofern auch in Theorie weit davon entfernt, „aus der Gesellschaft soziale Konflikte und damit die Politik selbst eliminieren zu wollen“, wie Forst $(2007,165)$ unterstreicht. In großen Bereichen des politischen Lebens ist demnach schlicht keine Einigung zu erwarten (Gališanka 2019, 197).

Der Realismus müsste eine radikalere These in Bezug auf die Reibungen, Dissonanzen und Unstimmigkeiten in der Politik vertreten und geriete dabei nahe an die Thesen Carl Schmitts (IBWD, 78). Es stimmt jedoch, dass sich Rawls in Theorie kaum mit jenen befasst, die die Grundidee der Gesellschaft als einem auf den Prinzipien der Reziprozität und Unparteilichkeit beruhenden Kooperationskontexts ablehnen. Denn das Frühwerk stützt sich auf die moralpsychologische Hypothese, dass die Neigungen zu ungerechtem Handeln von jenen, die in einer wohlgeordneten Gesellschaft aufwachsen und leben, „beseitigt oder jedenfalls unter Kontrolle gebracht werden“ können $(T G, 276)$. Rawls hofft, dass bei der „überwältigenden Mehrheit“ der Menschheit die Fähigkeit zu einem Gerechtigkeitssinn bestehe, der die Sicht auf das politische Leben beeinflusse (ebd., 425, 549).

Doch ist die Idee, dass eine wohlgeordnete Gesellschaft wohlgeordnete Subjekte hervorbringt (Honig 1993, 137), die ihre Natur als freie und gleiche Vernunftwesen ausdrücken, und sich dieses Regime deshalb nahezu straffrei stabilisiert, wohl durchaus als Wunschdenken zu kritisieren. Die Kongruenzthese, die Rawls mit der Kantischen Deutung des Urzustands verknüpft hatte, war unrealistisch $(T G, \S \S 40,86)$. Gleichwohl lässt sich mit ihm betonen, dass sich die politische Philosophie bedacht zu den Quellen und Formen von Konflikten als Ausgangspunkte und zur Möglichkeit ihrer 
Auflösung zu äußern hat. Dafür bedarf es eines Begriffs der Person, psychologischer Prämissen sowie einer Idee von für das Denken, Deliberieren und Urteilen günstigen Bedingungen. Es ist eine Stärke von Rawls, dass er stets verdeutlicht, aus welchen Quellen relevanter Dissens und Meinungsverschiedenheiten entspringen, während mir dies in vielen selbsterklärt realistischen Ansätzen oft schleierhaft bleibt: Woher die Gewissheit, dass bestimmte moralische Uneinigkeiten der politischen Gegenwart unüberwindbar sind? Einer pessimistischen Auffassung menschlicher Natur und der Grenzen rationaler Urteilskraft? Stützt sich der politische Realismus letztlich auf eine Position in der Metaethik, etwa einen Nonkognitivismus, der einen umfassenden Skeptizismus rechtfertigen könnte?

Eine politische Theorie, die gegebene Bedürfnisse, Interessen und Konflikte, etwa ob differierender Identitätskonzepte, ernstnimmt, läuft Gefahr, sich dem Widerstreit der Meinungen vorschnell zu beugen und ihnen einen zu großen Einfluss auf die Theoriebildung zu gestatten - dem gespaltenen Status quo würde eine Plausibilität unterstellt, die er vielleicht nicht verdient. Ohnehin führt kein Weg daran vorbei, sich bei abstrakten normativen Argumenten auf geteilte und möglichst schwache Grundprämissen zu stützen, weil die wesentliche Eigenschaft eines intersubjektiven Rechtfertigungsprozesses die stets revisionsfähige Übereinstimmung ist $(T G, 630)$. Dies bringt mich zum letzten Einwand, den ich hier diskutieren möchte: die mutmaßliche Ahistorizität der Gerechtigkeit als Fairness.

\section{Gerechtigkeitssinn und Geschichte}

Rawls geht davon aus, dass sich Regierende teils von ihren moralischen Überzeugungen leiten lassen. Da es keinem Verfassungssystem nur durch checks and balances gelingt, die Politik in Richtung eines gerechten Ergebnisses zu steuern, bedürfe es eines öffentlichen Gerechtigkeitssinns: „Es scheint also, daß eine richtige Theorie der Politik in einem konstitutionellen Regierungssystem eine Theorie der Gerechtigkeit voraussetzt, die den Einfluß moralischer Gesinnung auf das politische Geschehen erklärt“ ( $T G, 535)$. Es scheint jedoch auch, dass eine richtige Theorie der Politik der historischen Reflexion bedarf - ein Punkt, den Williams vor allem in seinem Spätwerk betont, in dem er sich oft auf Thukydides und Nietzsche stützt: „[P]olitische Philosophie erfordert Geschichte“ (IBWD, 53; Übers. A.U.).

Williams kritisiert, dass der moralistische Liberalismus eine zu idealisierte Auffassung bezüglich des kognitiven Status seiner Entstehung hat 
und es ihm an einer Irrtumstheorie mangelt (IBWD, 9). Doch ist ihm zufolge die Moderne durchaus eine Quelle der Normativität, aus der heraus sich die politische Realität in Frage stellen lässt, weil „unsere“ Vorstellungen von legitimen Antworten auf die erste politische Frage heute mit kontextuellem Inhalt angereichert sind. Angesichts historischer Entwicklungen wie der Säkularisierung, tiefgreifenden politischen Erfahrungen und der Entzauberung traditioneller Legitimierungen asymmetrischer Machtordnungen, - etwa als gottgewollt oder naturgegeben -, erscheinen uns aufgrund eines ausgeprägten „reflexiven Bewusstseins“ nur noch gewisse Rechtsverhältnisse als sinnvoll (IBWD, 11, 105; Williams 2011, 181). Wegen ihres „weltweiten Erfolgs“ ist laut Williams die Idee eines legitimen Staates eng mit der Idee einer partizipatorischen Demokratie verknüpft; das Konzept modernen Rechts berge ein progressives demokratisches Ideal (IBWD, 15).

Rawls argumentiert wiederum, dass die Gerechtigkeitsgrundsätze „nicht von bestehenden Bedürfnissen oder gesellschaftlichen Verhältnissen abhängen“ $(T G, 296)$. Seine Konzeption fußt auf allgemeinen Idealvorstellungen vom Menschen und der Gesellschaft, die zwar nicht apriorisch gerechtfertigt sind, uns aber einen archimedischen Punkt finden lassen, von dessen Warte aus die Politik kritisch beurteilt werden kann. Die Intuitionen und normativen Grundbegriffe, die die Konstruktion des Urzustands anleiten, sind entscheidend (Habermas 1996, 77f.). Ich habe oben erläutert, dass Rawls allgemeine Bedürfnisse nach Primärgütern zu rekonstruieren versucht und dass sich seine Theorie auf Ansichten über die vernünftigen Bedingungen für die rationale „Wahl erster Grundsätze“ beruft ( $T G$, 629). Der Einfluss der Geschichte jedoch auf die wohlüberlegten Urteile und Ideale bleibt in Theorie undeutlich. Doch sei bedacht, dass sich Rawls, wie erwähnt, auf Gerechtigkeitsvorstellungen verlässt, die den Parteien im Urzustand zur Auswahl stehen und „den historischen Konsensus darüber darstellen, welche Moralvorstellungen bisher als die vernünftigeren und praktikableren erscheinen“ (ebd., 631; Herv. A.U.). Rawls scheint davon auszugehen, dass sich demokratische Vorstellungen der Freiheit und Gleichheit in der Geschichte des politischen Denkens herausgebildet und bewährt haben. Ferner sagt er, dass der Vier-Stufen-Gang „durch die Verfassung der Vereinigten Staaten und ihre Geschichte nahegelegt“ werde (ebd., 224, Fn. 1). Einem Überlegungsgewicht nähert man sich nicht ohne historische Bezüge.

Der Gerechtigkeitssinn, den Rawls beschreibt, entwickelt sich unter den sozialen und politischen Bedingungen, die eine Geschichte haben. In 
Theorie stehen schließlich die erwähnten und nicht leicht zu deutenden Sätze, dass normative Grundsätze im Lichte bekannter Umstände des menschlichen Lebens gerechtfertigt sein und von der Eigenart dessen, was sie regulieren sollen, abhängen müssen. Die Tradition demokratischen Denkens und jene Ideale, die uns ob unserer sozialer Praktiken und der ihr inhärenten Normen als gerechtfertigt erscheinen und der Interpretation bedürfen, spielen eine entscheidende Rolle auch im frühen Argument (James 2005): „Bereits vor seiner politischen Wende ging Rawls von der liberal-demokratischen Welt als Ausgangspunkt aus - und richtete sein Werk an sie“ (Weithman 2010, 11; Übers. A.U.).

Dies führt zu einer wichtigen Erläuterung in Rawls' Dewey-Lectures, auf die ich kurz eingehen möchte, um den gedanklichen Faden des zweiten Abschnitts wieder aufzunehmen. Der Kern seines Konstruktivismus ist, wie er später argumentiert hat, eine Konzeption der moralischen Person, die idealiter in der öffentlichen Kultur einer demokratischen Gesellschaft wurzelt: „Das Ziel politischer Philosophie [...] ist es, diese gemeinsamen Begriffe und Grundsätze, von denen angenommen wird, daß sie im Common sense [sic!] schon latent vorhanden sind, zu artikulieren und explizit zu machen“ (Rawls 1992a, 84). Wie Williams geht es ihm um die emanzipatorischen Potenziale innerhalb moderner demokratischer Gesellschaften, in die Überzeugungen und Selbstverständnisse bereits diffundiert sind: „Was eine Gerechtigkeitskonzeption rechtfertigt“, schreibt er, „ist nicht ihr Wahrsein bezüglich einer vorgängigen, uns vorgegebenen Ordnung, sondern ihre Übereinstimmung mit einem tieferen Verständnis unserer selbst und unserer Bestrebungen, sowie unsere Einsicht, daß diese Lehre in Anbetracht unserer Geschichte und der in unser Leben eingebetteten Traditionen die vernünftigste für uns ist“ (ebd., 85; Herv. A.U.). Rawls meint hier nicht, dass die Vorstellung der freien und gleichen Person, die ihre praktische Vernunft benutzt, und das Ideal der Gesellschaft als kooperativem System zum gegenseitigen Vorteil aus der politischen Kultur einer Demokratie einfach und direkt herauspräpariert werden können (Forst 2017). Mein Punkt ist schlicht, dass sich seine Gerechtigkeitstheorie, gestützt auf eine bescheidene philosophische Anthropologie (Gališanka 2019, 182), durchaus für eine konstruktive Interpretation der politischen Gegenwart und ihrer Geschichte als Startpunkt öffnet, um eine nichtmetaphysische Grundlage festzuklopfen, die die Informationsbeschränkungen im Urzustand normativ zu rechtfertigen imstande ist. Aus diesem Grund glaubt Rawls, sagen zu können, dass die Bedingungen dieses hypothetischen Zustands „tatsächlich akzeptiert“ werden $(T G, 637)$. 
Nun findet sich in seinem Neuentwurf der Gerechtigkeit als Fairness in einer Fußnote die eminente Erkenntnis, dass die politische Philosophie stets missbraucht werden könne, „um einen ungerechten und unwürdigen Status quo zu rechtfertigen und somit im Marxschen Sinne ideologisch zu werden“ ( $G a F, 23$, Fn. 4). Darauf folgen knifflige Fragen, etwa, ob die Grundideen seiner Gerechtigkeitskonzeption eine Selbsttäuschung sein könnten. Rawls stellt sich selbst unter jenen Ideologieverdacht, den ich im zweiten Abschnitt kurz diskutiert habe. Im Lichte dieser Sorge und der in einigen aktuellen sozialwissenschaftlichen Studien hervorgehobenen Erosion repräsentativer Demokratien und der Krise des politischen Liberalismus ist eine Relektüre der Theorie und der darin gemachten Annahmen über den Gerechtigkeitssinn bedeutsam. Wer die Grundideen des Frühwerks weiter verteidigt, muss eine gute Antwort auf die Frage finden, die Rawls bezüglich des womöglich ideologischen Charakters seiner Denkfiguren in seinem Spätwerk aufwirft: „Wie können wir nachweisen, daß sie es nicht sind?“ (ebd.). ${ }^{10}$

\section{Im Schatten von Rawls?}

In diesem Aufsatz habe ich mich mit einigen prima facie gewichtigen Einwänden von Raymond Geuss und Bernard Williams gegen John Rawls' frühe Rechtfertigung der Gerechtigkeit als Fairness befasst. Dabei wurde deutlich, dass ihre Kritik, so spannend und erhellend sie bisweilen ist, nicht immer jene Durchschlagskraft hat, wie es zunächst scheint. Geuss und Williams neigen dazu, die interpretative Natur von Rawls' Ansatz, seine Anerkennung der Grenzen der Philosophie sowie die Tatsache herunterzuspielen, dass er in Theorie weder einfach moralische Prinzipien auf die politische Sphäre anwendet noch konflikt- oder machtblind ist. Der neue politische Realismus geriert sich als Bewegung des Widerstands gegen ein übertriebenes Moralisieren in der politischen Philosophie. Das ist zu begrüßen. Doch wäre es angebracht, diejenigen, gegen die man revoltiert, zunächst so ernst wie möglich zu nehmen, statt ein Theoriegebäude in Gänze einreißen zu wollen.

Geuss lehnt Rawls' Herangehensweise an die politische Philosophie vollends ab. Er sieht „schlicht keinen Anlass, tiefer in ein Gedankensystem einzusteigen, dessen Grundausrichtung er ohnehin als fehlgeleitet ansieht“

10 Großartige neuere Versuche, den politischen Liberalismus gegen den Ideologieverdacht und den Vorwurf einer Verdrängung praktischer Politik zu verteidigen, sind Möllers (2020) und Larmore (2020). 
(Freyenhagen und Schaub 2010, 468; OE, 29). Rawls' Vorstellung einer hermeneutischen Philosophie ist dieser ruppigen Herangehensweise wiederum diametral entgegengesetzt, sagt er doch in einer seiner Vorlesungen zur Geschichte der politischen Philosophie über Hobbes' Leviathan: „Wenn man einen so umfassenden und inhaltsreichen Text betrachtet und möglichst viel Nutzen daraus ziehen möchte, muß man ihn bei der Interpretation so stark und so interessant wie möglich machen. Widerlegungsversuche haben ebensowenig Sinn wie der Nachweis, daß sich der Autor irgendwie geirrt hat oder daß sein Argument nicht schlüssig ist. Man sollte das Bestmögliche daraus machen und ein Gefühl dafür entwickeln, in welche Richtung das Ganze gehen könnte, wenn man es möglichst überzeugend wiedergibt“" (GPP, 96f.).

Ähnlich sollte auch mit Theorie verfahren werden. Damit meine ich nicht, dass die Kritik, die politische Philosophie habe auch deshalb moralistische Tendenzen, weil sie noch im Schatten von Rawls' Gedankenfiguren stehe, nicht teils zuträfe oder dass der Realismus keine wichtigen Impulse setze. Doch darf der Vorwurf des Moralismus nicht zu schnell bei der Hand sein. Ludwig Wittgenstein, dessen Denken einen großen Einfluss auf Rawls ausgeübt hat (Gališanka 2019, 8), hat in den Philosophischen Untersuchungen schön gesagt, dass es stets leichter ist, „ein Problem zuzuschütten, als es zu lösen“ (Wittgenstein 2003, 182). Die Kritik der moralischen Kritik der Politik bedarf einer klaren normativen Grundlage. Zweifellos müssen wir heute in vielen Belangen über Rawls hinausgehen - vor allem über seinen gerechtigkeitstheoretischen Etatismus angesichts einer „politisch fragmentierte[n] Weltgesellschaft“ (Habermas 2019, 99). Ihn jedoch gänzlich hinter uns zu lassen, das ist eine unrealistische Bestrebung. Dafür stecken in seiner Theorie zu viele wertvolle Gedanken, etwa jene zur „Gegenseitigkeitsvorstellung“ oder zum „fairen Wert" der politischen Freiheit und dessen Gefährdung durch Wohlstandsungleichheit ( $T G, \S \S 17,36)$.

Wer einen politischen Anti-Moralismus verficht, sollte sich weiter auf ein produktives Gespräch auch mit dem frühen Rawls einlassen und die inneren Spannungen seines Egalitarismus beachten. Die Unterscheidung zwischen einer politischen und vor-politischen Moralität und allgemein die Idee der „Autonomie des Politischen" führen wiederum auf philosophisches Glatteis. Oft wird nicht ersichtlich, auf welche alternativen normativen Voraussetzungen und Rechtfertigungsstrategien sich der neue politische Realismus genau stützt. Diese Denkschule muss sich der Rückfrage stellen, inwiefern die Begründung mancher ihrer Thesen, - etwa bezüglich unauflöslicher politischer Uneinigkeiten, der verzerrenden Einflüsse der Macht 
auf moralische Urteile oder der historischen Kontingenz von Idealen wie der Menschenrechte oder Gerechtigkeit -, von metaethischer Beweislast befreit und empirisch evident sind.

Spöttische Kritik jedoch, die meint, auf genaue Lektüre verzichten zu können, führt auf Abwege. Ein Vorankommen in der politischen Philosophie gelingt, wenn wir die engen Grenzen jener großen Theorien, die wir kritisieren, zu weiten versuchen.

\section{Danksagung}

Für wertvolle Kritik, Hinweise und Korrekturen bedanke ich mich bei Felix Kämper, Andrea Klonschinski, Darrel Moellendorf, Elif Özmen, Martin Renz, Lukas Sparenborg und den anonymen Gutachter:innen der Zeitschrift für Praktische Philosophie.

\section{Literatur}

Buddeberg, Eva. 2020. „Wer kritisiert wen im Namen welcher Moral?“ In Kritik des Moralismus, herausgegeben von Christian Neuhäuser und Christian Seidel, 183205. Berlin: Suhrkamp.

Estlund, David. 2020. Utopophobia. On the Limits (If Any) of Political Philosophy. Princeton: Princeton University Press.

Forrester, Katrina. 2019. In the Shadow of Justice. Postwar Liberalism and the Remaking of Political Philosophy. Princeton: Princeton University Press.

Forst, Rainer. 2007. Das Recht auf Rechtfertigung. Elemente einer konstruktivistischen Theorie der Gerechtigkeit. Frankfurt am Main: Suhrkamp.

Forst, Rainer. 2017. „Political Liberalism: A Kantian View“. Ethics 128 (1): 123-144. https://doi.org/10.1086/692945.

Freyenhagen, Fabian, und Jörg Schaub. 2010. „Hat hier jemand gesagt, der Kaiser sei nackt? Eine Verteidigung der Geusschen Kritik an Rawls' idealtheoretischem Ansatz". Deutsche Zeitschrift für Philosophie 58 (3): 457-477. https://doi. org/10.1524/dzph.2010.58.3.457.

Gališanka, Andrius. 2019. John Rawls. The Path to a Theory of Justice. Cambridge/ MA: Harvard University Press.

Galston, William A. 2010. „Realism in Political Theory“. European Journal of Political Theory 9 (4): 385-411. https://doi.org/10.1177/1474885110374001.

Gledhill, James. 2012. „Rawls and Realism“. Social Theory and Practice 38 (1): 5582. https://doi.org/10.5840/soctheorpract20123813.

Geuss, Raymond. 2005. Outside Ethics. Princeton: Princeton University Press. 
Geuss, Raymond. 2010. „Realismus, Wunschdenken, Utopie“. Deutsche Zeitschrift für Philosophie 58 (3): 419-429. https://doi.org/10.1524/dzph.2010.58.3.419.

Geuss, Raymond. 2011. Kritik der politischen Philosophie. Eine Streitschrift. Hamburg: Hamburger Edition.

Geuss, Raymond. 2016. Reality and Its Dreams. Cambridge/MA: Harvard University Press.

Habermas, Jürgen. 1996. Die Einbeziehung des Anderen. Studien zur politischen Theorie. Frankfurt am Main: Suhrkamp.

Habermas, Jürgen. 2019. Auch eine Geschichte der Philosophie. Bd. 1: Die okzidentale Konstellation von Glauben und Wissen. Berlin: Suhrkamp.

Hampshire, Stuart. 2000. Justice Is Conflict. Princeton: Princeton University Press.

Hobbes, Thomas. 1984. Leviathan oder Stoff, Form und Gewalt eines kirchlichen und bürgerlichen Staates. Frankfurt am Main: Suhrkamp.

Honig, Bonnie. 1993. Political Theory and the Displacement of Politics. New York: Cornell University Press.

James, Aaron. 2005. „Constructing Justice for Existing Practice: Rawls and the Status Quo“. Philosophy \& Public Affairs 33 (3): 281-316. https://doi.org/10.1111/ j.1088-4963.2005.00034.x.

Jubb, Robert. 2014. „Playing Kant at the Court of King Arthur“. Political Studies 63 (4): 919-934. https://doi.org/10.1111/1467-9248.12132

Kersting, Wolfgang. 2001. John Rawls zur Einführung. Hamburg: Junius.

Larmore, Charles. 2020. What Is Political Philosophy? Princeton: Princeton University Press.

Leader Maynard, Jonathan, und Alex Worsnip. 2018. "Is There a Distinctively Political Normativity?” Ethics 128 (4), 756-787. https://doi.org/10.1086/697449.

Mason, Andrew. 2010. „Rawlsian Theory and the Circumstances of Politics“. Political Theory 38 (5): 658-683. https://doi.org/10.1177/0090591710372862.

McQueen, Alison. 2018. „The Case for Kinship: Classical Realism and Political Realism". In Politics Recovered: Realist Thought in Theory and Practice, herausgegeben von Matt Sleat, 243-269. New York: Columbia University Press.

Möllers, Christoph. 2020. Freiheitsgrade. Berlin: Suhrkamp.

Rawls, John. 1975. Eine Theorie der Gerechtigkeit. Frankfurt am Main: Suhrkamp.

Rawls, John. 1992a. „Kantischer Konstruktivismus in der Moraltheorie“. In Die Idee des politischen Liberalismus, herausgegeben von Wilfried Hinsch, 80-158. Frankfurt am Main: Suhrkamp.

Rawls, John. 1992b. „Der Gedanke eines übergreifenden Konsenses“. In Die Idee des politischen Liberalismus, herausgegeben von Wilfried Hinsch, 293-332. Frankfurt am Main: Suhrkamp. 
Rawls, John. 1998. Politischer Liberalismus. Frankfurt am Main: Suhrkamp.

Rawls, John. 2003. Gerechtigkeit als Fairneß. Ein Neuentwurf. Frankfurt am Main: Suhrkamp.

Rawls, John. 2008. Geschichte der politischen Philosophie. Frankfurt am Main: Suhrkamp.

Rossi, Enzo, und Matt Sleat. 2014. „Realism in Normative Political Theory“. Philosophy Compass 9 (10): 689-701. https://doi.org/10.1111/phc3.12148.

Rossi, Enzo. 2019. „Being Realistic and Demanding the Impossible“. Constellations 26 (4): 638-652. https://doi.org/10.1111/1467-8675.12446.

Sangiovanni, Andrea. 2008a. „Justice and the Priority of Politics to Morality“. The Journal of Political Philosophy 16 (2): 137-164. https://doi.org/10.1111/j.14679760.2007.00291.x.

Sangiovanni, Andrea. 2008b. „Normative Political Theory: A Flight from Reality?“. In Political Thought and International Relations. Variations on a Realist Theme, herausgegeben von Duncan Bell, 219-240. New York: Oxford University Press.

Sangiovanni, Andrea. 2014. „Scottish Constructivism and the Right to Justification“. In Justice, Democracy and the Right to Justification: Rainer Forst in Dialogue, herausgegeben von Rainer Forst, 29-64. London: Bloomsbury Publishing.

Sangiovanni, Andrea. 2016. „How Practices Matter“. The Journal of Political Philosophy 24 (1): 3-23. https://doi.org/10.1111/jopp.12056.

Schaub, Jörg. 2012. „Politische Theorie als angewandte Moralphilosophie? Die realistische Kritik“. Zeitschrift für Politische Theorie 3 (1): 8-24. https://www.budrich-journals.de/index.php/zpth/article/view/9663.

Sleat, Matt. 2013. Liberal Realism. A Realist Theory of Liberal Politics. Manchester: Manchester University Press.

Sleat, Matt. 2018. „Introduction. Politics Recovered - On the Revival of Realism in Contemporary Political Theory". In Politics Recovered. Realist Thought in Theory and Practice, herausgegeben von Matt Sleat, 1-25. New York: Columbia University Press.

Thomas, Alan. 2017. „Rawls and Political Realism: Realistic Utopianism or Judgment in Bad Faith?". European Journal of Political Theory 16 (3): 304-324. https:// doi.org/10.1177/1474885115578970.

Waldron, Jeremy. 1999. Law and Disagreement. Oxford: Oxford University Press.

Waldron, Jeremy. 2016. Political Political Theory. Essays on Institutions. Cambridge/MA: Harvard University Press.

Weithman, Paul. 2010. Why Political Liberalism? On John Rawls's Political Turn. Oxford: Oxford University Press.

Williams, Bernard. 2002. Truth and Truthfulness. An Essay in Genealogy. Princeton: Princeton University Press. 
Williams, Bernard. 2005. In the Beginning Was the Deed. Realism and Moralism in Political Argument. Princeton: Princeton University Press.

Williams, Bernard. 2011. Ethics and the Limits of Philosophy. Abingdon: Routledge.

Williams, Bernard. 2014. Essays and Reviews. 1959-2002. Princeton: Princeton University Press.

Wittgenstein, Ludwig. 2003. Philosophische Untersuchungen. Frankfurt am Main: Suhrkamp.

Wolin, Sheldon S. 2004. Politics and Vision. Continuity and Innovation in Western Political Thought. Expanded Edition. Princeton: Princeton University Press. 
